\section{Secret médical}

Quelle attitude du praticien quand des instances ou personnes
extérieures demandent des renseignements à propos d'un patient?

J. Martin, O. Guillod

\section{Préambule}

Le médecin cantonal ou le juriste spécialisé en la matière sont souvent interrogés à propos des questions médico-légales ou médico-éthiques liées au secret professionnel. Rappelons que, s'agissant des professions de la santé, secret médical et secret professionnel sont des notions identiques. En effet l'art. $321 \mathrm{du}$ Code pénal suisse (accessoirement l'art. 321 bis relatif aux recherches scientifiques avec les données médicales), qui traite de ce sujet, s'applique à plusieurs professions (dont les médecins, médecins-dentistes, pharmaciens, sages-femmes et leurs auxiliaires). On peut donc utiliser l'un et l'autre termes de manière interchangeable. On parle aussi, et c'est une terminologie pertinente, de secret du patient ("Patientengeheimnis" - voir article 11 de la version allemande du Code de déontologie de la Fédération des médecins suisses). ${ }^{1}$

Rappelons que, dans la doctrine et la pratique suisses, le secret médical n'a pas le caractère «intouchable», absolu (ou considéré comme tel) qu'il a par exemple en France. Cela est dû, légitimement à notre sens, au fait qu'il y a des circonstances où il est de l'intérêt d'autres personnes, voire d'intérêt public, de pouvoir faire état de circonstances et données couvertes par le secret médical.

Ainsi qu'on l'enseigne en médecine légale, il faut avoir à l'esprit les trois manières pour le professionnel d'être délié de ce secret:

1. Lorsque le patient lui-même le délie. C'est la règle d'or; chaque fois que cela est possible, c'est de cette manière qu'il convient d'être délié. En principe (sauf urgence), le médecin commencera toujours par poser la question à son malade avant d'envisager de s'adresser à l'autorité cantonale compétente (cf. 3 ci-dessous).

\section{Correspondance:}

Dr Jean Martin, privat-docent

Médecin cantonal

Service de la santé publique

Cité-Devant 11

CH-1014 Lausanne

Prof. Olivier Guillod

Université de Neuchâtel

Institut de droit de la santé

Avenue du $1^{\text {er }}$ Mars 26

CH-2000 Neuchâtel
2. Lorsqu'une disposition légale fédérale ou cantonale le délie. Selon les cas, soit il a alors la possibilité de parler des situations que précise la loi, soit il en a l'obligation.

3. Enfin, dans les autres cas, à savoir ceux où ni le patient ni la loi ne lève le secret, le médecin peut selon l'art. 321 CPS demander d'être délié par l'autorité supérieure ou de surveillance désignée dans chaque canton. Dans le canton de Vaud, il s'agit du Conseil de santé, qui fonctionne en pratique, pour la plupart des cas, par une délégation de deux membres.

N.B.: Le médecin est adéquatement délié quand une des conditions ci-dessus est remplie. Il n'est jamais nécessaire pour la même situation d'être délié du secret professionnel de deux ou plusieurs manières. Ainsi, si le secret est levé par le patient capable de discernement, le médecin est libre de parler (de ce dont le patient souhaite qu'il parle - et seulement de cela) et n'a besoin d'aucune confirmation complémentaire.

Il convient aussi de rappeler que les professionnels de la santé ne peuvent transmettre à des confrères des éléments du dossier d'un patient qu'avec l'accord de ce dernier.

1 Bien entendu, le secret de fonction est lui différent (art. 320 du Code pénal). Seuls les fonctionnaires (fédéraux, cantonaux et communaux), ainsi que les membres des autorités, y sont soumis. A noter aussi que la législation cantonale peut soumettre au secret de fonction les collaborateurs d'institutions de droit public. La délimitation entre secret professionnel (art. 321 CPS) et secret de fonction (art. 320 CPS) en ce qui concerne l'activité de médecins travaillant dans le secteur public fait encore objet de controverses juridiques. La thèse le plus souvent soutenue aujourd'hui affirme que le médecin est soumis au secret professionnel en ce qui concerne les informations relatives au patient et à son traitement et qu'il est en revanche soumis au secret de fonction en ce qui concerne les informations relatives à l'institution dans laquelle il travaille et au fonctionnement de celleci. Suivant cette thèse, le médecin doit donc être délié soit par le patient, soit par l'autorité supérieure, selon la nature des informations qu'il veut transmettre à un tiers. Par précaution, la pratique s'était toutefois instaurée (et est encore suivie) dans certains cantons de demander aux médecins du secteur public libérés du secret professionnel par le patient d'être au surplus déliés du secret de fonction par l'autorité administrative compétente (par exemple le conseiller d'Etat concerné dans le canton de Vaud) quand il s'agit d'informer des tribunaux, des services administratifs et d'autres tiers qui ne jouent pas de rôle lié au traitement du patient, et cela quelle que soit la nature des informations à communiquer. En revanche, le médecin occupé dans un service public n'a jamais besoin d'être délié du secret de fonction pour renseigner le malade lui-même ni, avec l'accord du patient, ses proches, d'autres intervenants du domaine des soins ou l'assureur prenant en charge le coût du traitement. Dans ces hypothèses, il s'agit à l'évidence du secret du patient ("Patientengeheimnis») puisque les données portent sur l'état de santé, les soins reçus ou d'autres éléments de la sphère intime du patient auxquels l'art. 320 CPS n'est pas applicable.

N.B.: C'est à tort qu'on entend occasionnellement des personnes engagées dans des associations privées parler de leur secret de fonction. Il s'agit alors plutôt d'un devoir de discrétion qui n'est pas en rapport avec le Code pénal, mais dépend le cas échéant du droit privé (Code des obligations) ou de la déontologie. 


\section{Quand le médecin est délié par son patient}

C'est donc la modalité majeure (la règle d'or), celle aussi qui est préférable déontologiquement. On peut rappeler ici la formule: le patient est le maître et le bénéficiaire du secret médical, le médecin en est le dépositaire. La relation médecin-malade est un contrat de mandat et le médecin doit à son mandant, entre autres choses, toutes informations en rapport avec la tâche qui lui a été confiée (prendre soin et si possible rétablir sa santé). C'est l'occasion de rappeler que le secret médical n'est en aucun cas opposable au patient lui-même. ${ }^{2}$

Dans le canton de Vaud, l'art. 80 de la loi sur la santé publique du 29 mai 1985 dispose «Les personnes qui exercent une profession mentionnée par l'article 321 du Code pénal suisse et qui relèvent de la présente loi sont liées par le secret professionnel. En raison de ce devoir de discrétion, elles ne peuvent être obligées de révéler les secrets qui leur ont été confiés ou dont elles ont eu connaissance dans l'exercice de leur profession, même si elles en sont déliées par l'intéressé ou par l'autorité compétente. Les avis et déclarations aux autorités sanitaires ordonnés par les lois et règlements font exception."

C'est là un privilège accordé aux professionnels concernés, dans la ligne d'une activité libérale. Quand nous avons (J. M.) à en parler avec des confrères, nous soulignons toutefois que le médecin doit avoir des raisons fortes, catégoriques, pour renoncer à informer (une personne ou instance extérieure) dans le cas où un patient capable de discernement le lui demande expressément. Fondamentalement, la primauté de la libre détermination du patient dans la relation médecin-malade n'est plus disputée aujourd'hui et ce n'est qu'exceptionnellement que le thérapeute pourrait être déontologiquement légitimé à refuser de parler alors que le patient lui est convaincu que c'est dans son intérêt. On ne saurait faire qu'un usage tout à fait limité de cette éventualité.

2 Dans plusieurs législations cantonales depuis une quinzaine d'années, on a donné un ancrage au devoir d'information du médecin, qui n'est donc pas un rôle facultatif. L'insuffisance de l'information du patient (préalable à la prestation), ou le fait de ne pas avoir demandé adéquatement son consentement libre et éclairé, peuvent être constitutifs de violation des règles de l'art (malpractice). Cela est bien admis aujourd'hui dans les pays occidentaux; on sait à cet égard qu'une importante proportion des plaintes formulées à l'endroit de thérapeutes sont en rapport avec l'information et le consentement (voir aussi art. $10 \mathrm{du}$ Code de déontologie de la FMH).

En ce qui concerne une éventuelle limitation de l'information du patient (parce qu'on estime qu'elle pourrait perturber/aggraver sa situation de santé), il ne s'agit pas d'une problématique liée au secret médical (qui n'est pas opposable au malade, une fois encore), mais au "privilège thérapeutique», qu'on devrait dénommer plutôt exception thérapeutique: voir à ce propos l'avis du Prof. W. Wiegand dans H. Honsell (Hrsg.), Handbuch des Arztrechts, Zürich, 1994. La tendance actuelle est de (beaucoup) restreindre cette exception. De plus, indépendamment de l'aspect juridique, l'expérience des dernières décennies a montré que, souvent, les patients acceptent et supportent la vérité beaucoup mieux qu'on aurait pu le penser, et sont plutôt rassérénés par elle.
Il convient de relever encore que, si le médecin est délié par l'autorité cantonale compétente, c'est qu'il a lui-même fait la demande et il paraît contradictoire qu'il se taise ensuite, encore qu'on puisse l'admettre (puisqu'il n'a obtenu qu'une autorisation de parler sans violer le secret professionnel). Surtout, on ne voit guère comment justifier le refus de parler quand le médecin est délié par le patient. La demande de ce dernier s'analyse juridiquement d'une part comme une renonciation à la protection de l'article 321 CPS (en ce sens, ce n'est effectivement qu'une autorisation de parler et non une obligation), mais d'autre part comme un mandat donné au médecin de communiquer certains renseignements à un tiers. Dès lors, ou bien le médecin suit les instructions que lui donne son mandant, comme il y est tenu par le contrat de mandat (art. 397 CO), ou bien il refuse la requête du patient et devrait alors résilier le mandat. Dans ce dernier cas, le patient pourra demander l'intégralité du dossier médical et transmettre lui-même les informations au tiers. Sauf situation d'exception, le médecin traitant devrait donc respecter la demande du patient, de manière aussi à pouvoir éclaircir les éventuelles obscurités du dossier ou en tout cas expliquer son contenu.

Dans d'éventuelles situations critiques, la première règle sera d'en discuter de manière approfondie, le médecin expliquant au patient pourquoi il lui paraît préférable de ne pas parler - ou le cas échéant de donner vers l'extérieur des renseignements sur certains sujets seulement et pas sur d'autres (ce qui est possible, sous réserve que cette sélection ne corresponde pas d'une manière ou de l'autre à un mensonge). Le médecin s'assure aussi que le patient connaît l'identité de celui qui demande des renseignements comme le contexte de la requête, et se met d'accord avec lui sur le contenu des révélations qu'il est autorisé à faire.

\section{Quand la loi dit que le médecin doit parler}

Ces situations sont en rapport notamment avec la loi fédérale sur la lutte contre les maladies transmissibles de l'homme (loi sur les épidémies) du 18 décembre 1970 et ses ordonnances d'application. Le médecin doit de routine adresser des informations au médecin cantonal, qui transmet à l'Office fédéral de la santé publique (annonce nominative pour certaines des affections indiquées dans les deux ordonnances sur les déclarations du 13 janvier 1999 - celles justifiant des mesures directes relatives à l'entourage ou à la santé publique).

D'autres obligations analogues peuvent exister en droit cantonal: dans le canton de Vaud, la loi sur la protection de la jeunesse du 29 novembre 1978 prévoit à son article 4 que les médecins ont le devoir d'annoncer tous mauvais traitements de mineurs (jusqu'à l'âge de 18 ans). Il ne s'agit donc pas seulement d'une possibilité. Cet article 4 paraît toutefois discutable ( 0 . G.) par rapport au principe de la force dérogatoire du droit fédéral, voulant qu'une disposition cantonale 
s'efface devant une disposition fédérale contraire. Or, l'art. 358ter CPS (cf. ci-dessous) prévoit la possibilité et non l'obligation pour les médecins d'alerter l'autorité tutélaire (dont les membres doivent de par leur fonction avertir aussi la justice pénale s'il y a délit).

D'autres cantons connaissent une obligation du médecin d'annoncer les crimes graves (homicide, par ex.) dont il aurait connaissance dans son activité.

\section{Quand la loi dit que le médecin peut parler}

Certains textes établissent la faculté/possibilité pour le praticien de parler mais ne l'y obligent pas. C'est le cas de la loi fédérale sur la circulation routière, en vue d' avertir les autorités concernées que la capacité de conduire d'une personne est mise en cause pour des raisons de santé ${ }^{3}$. La loi fédérale sur les stupéfiants et les substances psychotropes inclut, mutatis mutandis, une disposition comparable ${ }^{4}$. La loi vaudoise sur la santé publique prévoit à son art. 54 que les professionnels de la santé peuvent, dans les cas d'abus graves de médicaments ou de substances nocives, avertir le médecin cantonal.

L'art. $358^{\text {ter }}$ du Code pénal dispose: «Lorsqu'il y va de l'intérêt des mineurs, les personnes astreintes au secret professionnel ou au secret de fonction (art. 320 et 321) peuvent aviser l'autorité tutélaire des infractions commises à l'encontre de ceux-ci.» Cela vaut en particulier en cas de mauvais traitements.

Une fois encore, dans les cas ressortissant à cette section et la précédente, le médecin est donc délié d'office (automatiquement) en vue de la communication prévue par la loi.

\section{Quand il y a lieu de s'adresser à l'autorité supérieure ou de surveillance désignée dans chaque canton}

Cette autorité a pour mandat de recevoir les requêtes (le cas échéant les questions qui ne sont pas encore des requêtes) de professionnels soumis à l'art. 321 CPS et qui souhaitent transmettre des informations, dans des cas où:

- il n'est pas possible d'obtenir le consentement du patient (parce qu'il est dans le coma ou décédé ${ }^{5}$, ou que son état mental est déficient, par exemple), ${ }^{6}$ ou

- le patient refuse de délier le médecin, alors que ce dernier estime que des considérations liées à l'intérêt d'autres personnes sont d'un poids tel qu'il n'est pas possible d'obtempérer à ce refus,

- et où aucune disposition législative fédérale ou cantonale ne délie d'office le médecin dans la situation en cause (cf. supra).

Les instances chargées de cette tâche diffèrent selon les cantons (cela peut être le médecin cantonal ou le Chef du département chargé de la santé publique, c'est dans le canton de Vaud le Conseil de santé). Il n'est pas rare que nous ayons (J. M.) une première conversation téléphonique (appel du professionnel détenteur du secret) qui permet de discuter - anonymement - d'éventuels aspects spécifiques du cas et des enjeux y relatifs. Nous demandons toujours que la requête soit faite/confirmée par écrit. L'art. $321 \mathrm{du}$ Code pénal précise que l'accord de l'autorité supérieure doit lui aussi être donné par écrit.

Certaines situations posent des questions médicoéthiques, de conscience, délicates. Dans tous les cas, c'est le professionnel détenteur du secret qui décide finalement de demander à être délié ou pas, même s'il s'est appuyé pour prendre sa détermination sur l'avis de confrères ou d'autres personnes de bon conseil (ce qu'il peut faire auprès de n'importe qui sous réserve de présenter la situation de manière anonyme).

La décision de délier dépend alors de la pesée des intérêts en cause, la règle étant que l'autorisation est donnée lorsque l'intérêt à la divulgation l'emporte sur celui du maintien du secret:

- Celui du patient, bien entendu, auquel le médecin doit une attention diligente. Il reste que dans certaines circonstances l'appréciation de la situation amène à la conclusion que l'intérêt de tierces personnes (par exemple au sein de la famille) voire l'intérêt public peuvent ou doivent prévaloir sur la confidentialité liée au secret professionnel.

3 Art. 14, al. 4, de la LCR: «Tout médecin peut signaler à l'autorité de surveillance des médecins ainsi qu'à l'autorité compétente pour délivrer ou retirer les permis de conduire les personnes qui ne sont pas capables de conduire avec sûreté un véhicule automobile en raison de maladies ou d'infirmités physiques ou mentales ou pour cause de toxicomanie."

4 Art. 15, al. 1, de la LStup: «Les services administratifs, les médecins et les pharmaciens sont autorisés à signaler à l'autorité protectrice compétente ou à une institution de traitement ou d'assistance agréée les cas d'abus de stupéfiants qu'ils constatent dans l'exercice de leur activité officielle ou professionnelle, lorsqu'ils estiment que des mesures de protection sont indiquées dans l'intérêt du patient, de ses proches ou de la communauté.»

5 L'intérêt à la préservation du secret perdure après la mort du patient: le cas échéant le médecin doit être délié par l'autorité compétente avant de pouvoir donner aux proches ou aux héritiers accès au dossier médical du patient décédé (on notera à ce sujet que l'article 1 de l'Ordonnance fédérale sur la protection des données semble donner un droit d'accès aux proches, mais la légalité de cette disposition est discutable). De plus, dans les cas de cette information aux proches, on sera particulièrement attentif aux critères de pertinence et de proportionnalité des renseignements fournis.

6 Certaines distinctions sont appropriées en ce qui concerne l'hypothèse où le patient ne peut pas lui-même consentir. S'il est incapable de discernement (petit enfant, coma, déficience mentale grave), il convient d'abord de se demander s'il y a un représentant légal (père, mère, tuteur) qui pourrait donner valablement un consentement à la place du patient. Il faut aussi se demander si le patient (ex.: maladie d'Alzheimer) n'a pas donné au préalable procuration à un représentant privé de prendre les décisions de soins qui s'imposent, d'accéder à son dossier et, plus généralement, de défendre au mieux ses intérêts dans ce domaine. S'il existe un tel représentant, il peut autoriser l'éventuelle transmission d'informations à un ou des tiers. Qu'il soit légal ou privé, le représentant devrait se prononcer en priorité, et l'autorité cantonale compétente ne devrait pas intervenir sans s'assurer qu'on a recherché préalablement l'accord de ce représentant. 
- Il se peut que, alors qu'il a demandé d'être délié, le médecin se voie opposer un refus par l'autorité supérieure, qui estime que les arguments apportés ne sont pas déterminants. Dans la grande majorité des requêtes toutefois, dans notre expérience, la délégation du Conseil de santé estime que le médecin a évalué la situation en son âme et conscience; et ce n'est que pour des raisons fortes qui pourraient avoir échappé au praticien, ou si on ne parvient pas à voir quel intérêt prépondérant serait légitimement servi par le fait de révéler le secret, qu'elle refuse de délier ${ }^{7}$.

Dans différents cantons, l'instance cantonale compétente donne aussi, quand cela est possible, l'occasion aux patients de prendre position avant qu'elle se détermine sur la demande du médecin. Cela signifie que la demande d'être délié doit inclure l'identité du patient. Dans le cas où une information devrait, selon l'opinion du médecin, être transmise à un tiers et que le patient ne donne pas son accord, il est judicieux en règle générale que le thérapeute informe le malade sur la modalité de levée du secret par l'autorité cantonale qu'il pourrait utiliser (selon l'art. $321 \mathrm{CPS}$, al. 2). En effet, dans une telle situation, le patient peut finalement préférer délier lui-même le médecin plutôt que de donner lieu à une démarche auprès de l'autorité cantonale.

Cette manière de faire n'est naturellement pas envisageable dans des cas où le médecin apprend des faits graves pénalement punissables, par exemple patient meurtrier ou qui formulerait (ainsi que nous l'avons vécu dans notre propre expérience - J.M.) des menaces de mort crédibles à l'endroit de sa/son partenaire.

\section{Quand le médecin délié s'exprime par écrit}

La situation ne pose généralement guère de problème, le professionnel ayant alors le loisir de tourner sa plume dans l'encrier aussi précautionneusement qu'il le veut, en fonction du souhait du maître du secret, à savoir son malade, quand c'est celui-ci qui l'a délié. Il fera rapport de manière véridique et compréhensible. En principe, il donnera connaissance de ce rapport au patient lui-même, dans un esprit de transparence. Commentaire à cet égard d'un médecin légiste: «Je conseille toujours aux médecins de délivrer au patient le document réclamé par un tiers, charge à lui de le transmettre directement à ce dernier; ce qui évite toute contestation pour autant que le patient puisse exercer son contrôle sur le contenu du docu-

7 Par révélation, il faut entendre non seulement une communication orale ou écrite, mais également la remise de documents ou d'autres objets, même à un confrère médecin.

8 Nous avons pour principe de dire qu'on a le droit de poser n'importe quelle question à n'importe qui (y compris à un président de tribunal), sans garantie toutefois que la réponse soit toujours positive. ment médical, c'est-à-dire que celui-ci soit rédigé de façon compréhensible pour un profane» (P. Mangin; communication personnelle).

Si c'est la loi qui demande que le médecin annonce une situation, il le fera selon les dispositions applicables. S'il a été délié par l'autorité supérieure, dans un cas donc où le patient n'a pas pu ou pas voulu le délier, le médecin fournira les indications nécessaires et suffisantes en rapport avec la préoccupation qu'il avait en requérant la levée du secret.

\section{Quand le médecin délié s'exprime oralement}

Il convient d'abord de bien distinguer l'hypothèse où le médecin s'exprime comme témoin de celle où il s'exprime comme partie à une procédure judiciaire. Les considérations qui suivent se limitent au cas du témoignage.

Nous observons que c'est une situation que les médecins n'apprécient que modérément (d'autant moins qu'elle est rare pour eux) et qui suscite une certaine appréhension. Souvent, on nous consulte en demandant quelle attitude avoir, s'il est possible de connaître les questions à l'avance ${ }^{8}$, si on peut refuser de répondre, etc.

Même si on souhaite que l'évolution de la société n'amène pas ce type d'exercice à devenir une tâche trop fréquente des médecins, il n'y a pas lieu de s'émouvoir indûment quand on doit le faire. On gardera à l'esprit que, dans la mesure où de tels témoignages servent des intérêts qui doivent légitimement être préservés, ils sont utiles et nécessaires. Il convient d'observer quelques principes (voir section suivante) et d'avoir en mémoire le mandat reçu du patient (quand c'est lui qui a souhaité qu'on parle), respectivement la raison qui est apparue suffisamment forte pour que le professionnel demande de son propre chef la levée du secret. A ce propos, voir les considérations que nous émettons plus haut en rapport avec l'art. 80 de la loi vaudoise sur la santé publique.

Remarque à ce propos de M. Hp. Kuhn, juriste à la FMH: La question de savoir si le médecin délié du secret professionnel est tenu de parler est, dans les lois de procédure civile et pénale des cantons, réglée de manières diverses et, plus que cela, pas toujours clairement. Dans le cas où le médecin est délié mais qu'il est par ailleurs convaincu qu'il devrait néanmoins refuser de parler, il conviendra par conséquent d'examiner la question d'un devoir de parler, dans le cas particulier, en fonction de la législation cantonale pertinente.

\section{Quelques recommandations et principes}

Au risque d'être pédant, qu'on nous permette de relever des éléments qui peuvent servir de guide:

- D'abord, ne jamais mentir (et ne jamais inventer - si on ne sait pas, ou si on ne sait plus, admettre son ignorance). C'est une exigence juridique mais aussi morale, vis-à-vis de soi-même et de son 
patient. Dans des cas particulièrement difficiles existe la possibilité de décliner de répondre - sauf si une disposition légale cantonale ou fédérale impose au médecin de renseigner.

- Garder à l'esprit l'intérêt de son patient, en accordant le poids voulu au fait que, en tant que personne douée de discernement, c'est lui qui en principe sait le mieux ce qui est bon pour lui. S'il y a lieu de lui faire part de réserves à l'endroit des effets indésirables voire préjudiciables d'un témoignage du médecin, le faire avant de rédiger le rapport demandé ou l'audience du tribunal. Le médecin s'attachera alors à expliquer l'enjeu lié à l'information qu'il peut donner, expliquant en quoi elle consistera afin que le patient puisse se déterminer en toute connaissance de cause quant à l'opportunité ou non que son thérapeute parle.

- Donner une attention précise aux questions posées, qu'elles soient écrites ou orales (être sûr qu'on les a bien comprises) et renseigner sur ce qui est demandé. Eviter de se lancer dans des explications indûment prolixes, susceptibles de moins bien servir les intérêts en cause qu'une explication concise. Par ailleurs, s'il devait apparaître au médecin qui fournit un témoignage ou un rapport qu'il est important de signaler des éléments qui ne sont pas inclus dans les questions posées, il peut le faire (à condition que l'information qu'il apporte soit en rapport logique avec la situation pour laquelle le patient ou l'autorité compétente ont levé le secret).

- Rappelons le principe général de proportionnalité, essentiel en matière de protection des données: il ne faut donner que des informations pertinentes par rapport au but de la communication.

- Dans la mesure du possible, s'en tenir aux faits, aux éléments objectivement documentés. Il est vrai par ailleurs que des témoignages de médecins sous forme d'appréciations sont parfois souhaitables. Les formuler avec pondération.

- Etre attentif aux intérêts des tiers. C'est une préoccupation importante (qui vaut aussi quand le patient demande accès à son dossier). On ne saurait impliquer nommément des tierces personnes sans leur accord si l'information donnée peut d'une manière ou de l'autre les mettre en difficulté ou les faire apparaître sous un jour défavorable. Cela étant, il est en général possible de renseigner adéquatement à propos d'un patient en laissant dans l'anonymat les personnes ou intérêts des tiers qui n'ont pas à être nommés.

- Ne pas se laisser mettre sous pression par des demandes instantes. Cela peut se produire lors d'un témoignage oral, mais aussi si on est requis de manière urgente d'établir un document écrit. Sauf exception, on a le droit de demander un moment de réflexion ou d'indiquer qu'on répondra ultérieurement.

9 N.B.: La même chose vaut pour la personne sous tutelle, quand elle a son discernement en ce qui concerne des soins/traitements médicaux, y compris interventions chirurgicales.

\section{En cas de force majeure}

Le secret professionnel est une valeur forte dans nos sociétés, preuve en est que sa violation est sanctionnée par le Code pénal. S'agissant de le lever, il donne lieu aux dispositions discutées ci-dessus. Au surplus, il est judicieux de mentionner l'existence de l'art. 34 CPS, intitulé «Etat de nécessité», qui dispose notamment:

«1. Lorsqu'un acte aura été commis pour préserver d'un danger imminent et impossible à détourner autrement un bien appartenant à l'auteur de l'acte, notamment la vie, l'intégrité corporelle, la liberté, l'honneur, le patrimoine, cet acte ne sera pas punissable si le danger n'était pas imputable à une faute de son auteur et si, dans les circonstances où l'acte a été commis, le sacrifice du bien menacé ne pouvait être raisonnablement exigé de l'auteur de l'acte.

Si le danger était imputable à une faute de ce dernier ou si, dans les circonstances où l'acte a été commis, le sacrifice du bien menacé pouvait être raisonnablement exigé de l'auteur de l'acte, le juge atténuera librement la peine (art. 66).

2. Lorsqu'un acte aura été commis pour préserver d'un danger imminent et impossible à détourner autrement un bien appartenant à autrui, notamment la vie, l'intégrité corporelle, la liberté, l'honneur, le patrimoine, cet acte ne sera pas punissable. Si l'auteur pouvait se rendre compte que le sacrifice du bien menacé pouvait être raisonnablement exigé de celui auquel le bien appartenait, le juge atténuera librement la peine (art. 66).»

En cas d'urgence, quand des biens majeurs comme la vie ou la santé demandent à être sauvegardés sans aucun délai, cet article donne le droit de faire des choses en soi illicites; à condition que le but recherché ne puisse être atteint autrement et que l'action soit proportionnée au danger qu'on entend éviter. Ainsi, en situation de nécessité, le médecin peut exceptionnellement fournir à une personne ou une instance concernée des informations couvertes par le secret médical sans être pour autant sanctionnable, charge à lui de se faire délier formellement ensuite, dans le meilleur délai, par son patient ou par l'autorité cantonale compétente.

\section{A propos de capacité de discernement et de confidentialité liée au secret médical}

Il est important de savoir que la doctrine admise en Suisse est que le droit strictement personnel de demander des soins médicaux peut être exercé librement par la personne douée de discernement, y compris si elle est mineure ${ }^{9}$. L'âge auquel on acquiert cette capacité n'est pas fixé par la loi mais est une question d'appréciation: fondamentalement, il s'agit de savoir si, en ce qui concerne la question posée, l'adolescent est en mesure de juger adéquatement la situation et de décider sur la base de sa propre appréciation de ce qu'est son intérêt personnel. S'agissant de demander et recevoir des soins, le cas échéant les 
refuser, on peut admettre que cet âge est aux alentours de quatorze ans.

La conséquence importante est que le jeune peut demander au médecin d'observer la confidentialité à l'endroit de ses parents (on pense par exemple à des requêtes de contraception ou d'interruption de grossesse); il ou elle peut donc requérir un traitement médical à l'insu ou même contre le gré de ces derniers (qui pourtant, ayant la charge de leur enfant, restent débiteurs des frais médicaux ...). S’il juge ne pas pouvoir en conscience donner des soins à l'insu des parents, le médecin a le droit de décliner de le faire mais, d'un point de vue déontologique, doit s'assurer que la/le jeune a la possibilité sans difficulté indue de consulter un confrère qui accepte de la/le suivre, et pour autant que les soins ne soient pas nécessaires à court terme (urgence).

Entre autres choses, ce qui précède signifie que le médecin est valablement délié du secret médical par son patient mineur doué de discernement.

\section{Rappel à propos du rôle de médecin expert}

Au terme de cette discussion, il est approprié de rappeler que les questions se posent différemment quand on est chargé d'une expertise. En effet, le principe est alors que l'autorité compétente (dans un domaine donné), qui requiert pour se déterminer l'expertise d'un justiciable, donne un mandat correspondant au médecin (et, comme toujours lors d'expertises, dans quelque secteur que ce soit, c'est au mandant que l'expert rend son rapport). La personne examinée n'est pas alors, vis-à-vis du médecin, dans la situation du malade qui confie un mandat de soins au thérapeute de son choix. Typiquement, l'expert n'est pas choisi par le patient. Si dans certains cas il peut l'être de concert entre la personne expertisée et le mandant, la décision finale est du ressort de l'autorité.

On peut soutenir que l'expert médecin reste tenu au secret professionnel en vertu de l'article 321 CPS, qui ne pose pas de conditions personnelles spéciales d'assujettissement et ne différencie pas entre médecin thérapeute et médecin expert. Au sens large, le rôle d'expert peut être considéré comme entrant dans son activité professionnelle même si, à la différence de la situation normale, il n'y a pas le même rapport de confiance. Dans cette conception, tout ce que le médecin apprend à cette occasion serait protégé par l'art. 321 CPS. Selon une autre conception, plus fréquemment défendue, le médecin expert mandaté par un tribunal acquiert la qualité de fonctionnaire et est donc tenu à la confidentialité en vertu du secret de fonction de l'article 320 du Code pénal. Quoi qu'il en soit, le mandat d'expertise reçu du tribunal ou de l'autorité constitue, vis-à-vis de ces derniers, une exception au devoir de secret. Le médecin doit faire rapport au tribunal et, ce faisant, ne viole pas l'article 320 ni l'article 321 CPS. Il est évident en revanche qu'il ne peut pas informer qui que ce soit d'autre sur ce qu'il a constaté chez le patient en effectuant l'expertise. S’il le faisait, il serait punissable.

Ce qui importe du point de vue médico-éthique (cf. art. 6 du Code de déontologie de la FMH), c'est que la personne examinée soit consciente du statut particulier de l'expertise et du fait que le médecin doit fournir au mandant un rapport objectif, scientifiquement fondé, au mieux de ses connaissances et des observations qu'il aura pu faire, et ceci indépendamment des conséquences favorables, indifférentes ou défavorables que cela pourra avoir pour l'expertisé. L'expert n'est en aucune manière autorisé à cacher au mandant des éléments pertinents; si pour des raisons de conscience ou autres il se sentait contraint ou poussé à le faire, la seule attitude adéquate est de renoncer à l'expertise.

Dans la pratique, il y a des situations où le médecin traitant est sollicité pour donner une appréciation qui est du registre de l'expertise; c'est souvent le cas pour les examens périodiques de conducteurs ou pour des rapports destinés à l'autorité tutélaire. C'est admissible, étant entendu que le praticien a donc la responsabilité d'informer la personne qu'on n'est pas dans la relation usuelle patient-médecin traitant et qu'il est tenu de renseigner le mandant (Service des automobiles, Justice de Paix) de tout ce qu'il sait ou observe en rapport avec les questions posées, même si cela est défavorable.

\section{Références}

- Bertrand D, Harding TW, Mandofia Berney M, Ummel M. Médecine et droit médical. Genève: Editions Médecine $\&$ Hygiène; 1998.

- FMH. Vademecum du médecin suisse. Chiffre 33. Le secret médical. $5^{\mathrm{e}}$ édition. Berne: FMH, 1992.

- FMH. Code de déontologie. Bull Méd Suisses 1997;78:384-93. (Deutscher Text: Schweiz Ärztezeitung 1997:78:373-83)

- FMH. Site Internet www.fmh.ch; Droit; Recherches

- Guillod 0. Le secret médical, aujourd'hui. Dans: Le secret. Ethique, transparence et confidentialité. Cahiers ERIE, Université de Lausanne, 1996; p. 49-67.

Deux contributions parues récemment et basées sur notre expérience de médecin cantonal dans les relations avec des confrères, des patients et diverses instances et autorités, donnent des éclairages complémentaires sur des aspects éthiques évoqués ci-dessus:

- Martin J. Quand il s'agit d'apprécier, d'arbitrer, de conseiller - Expérience d'un médecin cantonal. Bull Méd Suisses 1998; 79:480-3.

- Martin J. La médecine, c'est communiquer - De l'importance de parler avec le patient, même à propos de questions peu agréables. Bull Méd Suisses 1999;80:282-4. 